\title{
New analytical Solutions of (3+1)-dimensional Shallow Water Wave equation (SWW)
}

\author{
Anjali Verma', Amit Verma ${ }^{2}$ \\ ${ }^{1}$ Assistant ProfessorUniversity Centre for Research and DevelopmentChandigarh University, Gharuan, Mohali, \\ Punjab-140413, India \\ ${ }^{2}$ Assistant ProfessorUniversity Centre for Research and DevelopmentChandigarh University, Gharuan, Mohali, \\ Punjab-140413, India
}

Article History: Received: 11 January 2021; Accepted: 27 February 2021; Published online: 5 April 2021

\begin{abstract}
In this paper, we have obtained new analytical solutions of (3+1)-dimensional SWW equation with Kudryashov method. The study of Shallow water wave equation plays an imperative role in wave theory. For calculation software Maple is used. The solutions obtained by this method are new.
\end{abstract}

Keywords: (3+1)-dimensional SWW equation, Kudryashov method, Analytical solutions.

\section{Introduction}

Waves in different parts of oceans are referred to by different names. Those differences are dependent on wave length. A shallow water wave is one that occurs at depths shallower than wave length of wave divided by 20. The $(3+1)$-dimensional SWW is

$$
v_{x x x x}+3 v_{x x} v_{y}+3 v_{x} v_{x y}-v_{y t}-v_{x z}=0
$$

Many researchers have obtained exact and numerical solutions of SWW equation. Peter A. Clarkson et al. [8] studied symmetry reduction and accurate solutions of SWW equation. B. Aydm et al. [4] obtained hodograph transform solutions of one dimensional nonlinear SWW equation by using integral transform techniques.

A.A Imani et al. [2] use reform of variation iteration method for computing coupled WhithamBroer-Kaup shallow water. Elizabeth L. Mansfield et al. [6] used classical and non classical reduction for (2+1)dimensional SWW equation. N.A. Matskevich et al. [7] present a few approaches to solve problems of shallow water oscillation in parabolic basin. Wiiliam Carlisle [9] find analytical solutions correspond to time dependent motions in parabolic basins. Ahmet et al. [3] obtained analytical solution for $(2+1)$ and $(3+1)$-dimensional SWW equation by using $\left(\mathrm{G}^{\prime} / \mathrm{G}\right)$ expansion method. Bijan et al. [5] used an extended elliptic function method to find solution of generalized shallow water wave equation. Anjali Verma et al. [1] used $\left(\mathrm{G}^{\prime} / \mathrm{G}\right)$ expansion method to discover traveling wave solutions of SWW equation.

\section{Methodology}

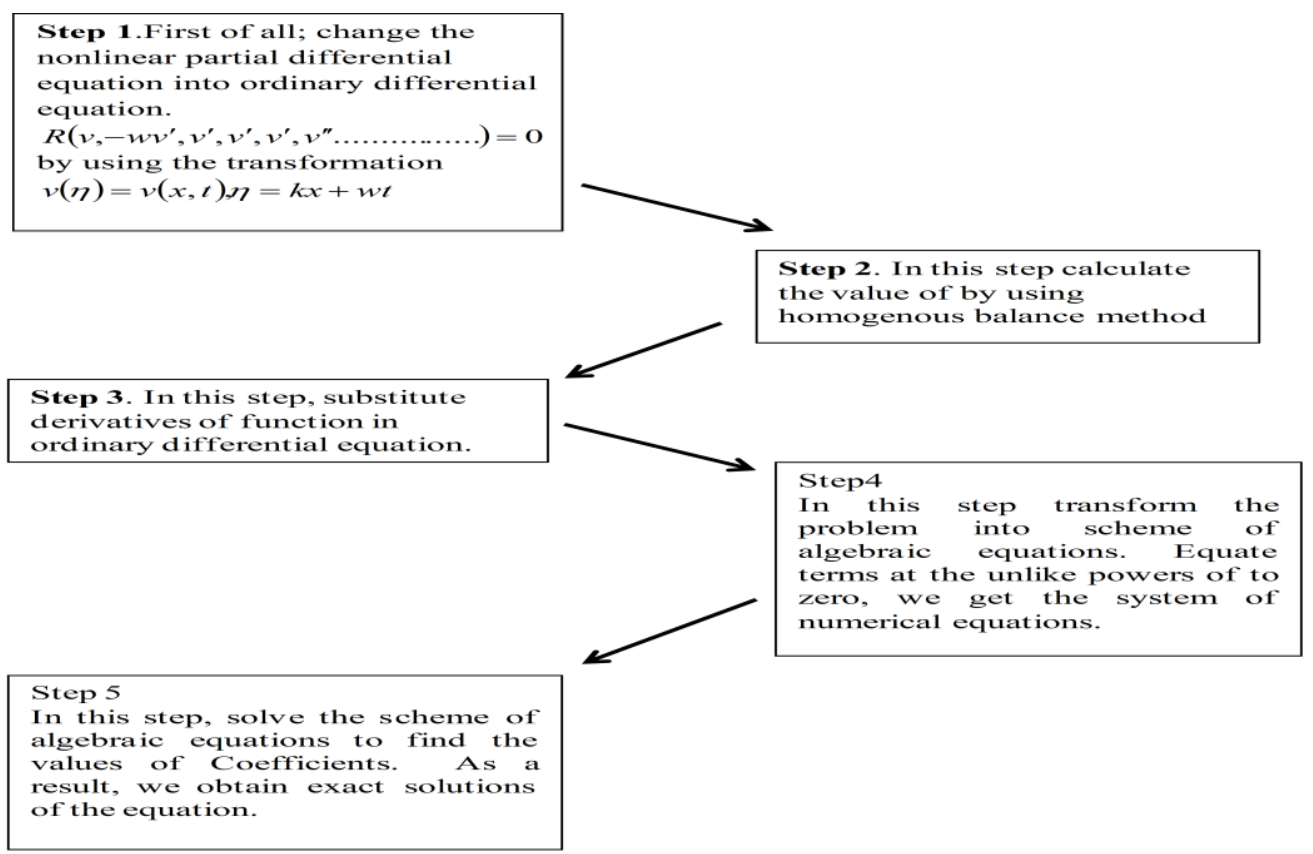




\section{Application of the Method}

Consider the application of the method for finding analytical solutions of (3+1)-dimensionl SWW equation

$$
v_{x x x x}+3 v_{x x} v_{y}+3 v_{x} v_{x y}-v_{y t}-v_{x z}=0
$$

In order to attain the solutions of equation (3.1), we make alteration

$$
v(x, y, z, t)=v(\eta), \eta=x+y+z-c t
$$

By using this transformation, we have obtained ODE

$$
v^{\prime \prime \prime \prime}+3 v^{\prime} v^{\prime \prime}+3 v^{\prime} v^{\prime \prime}+c v^{\prime \prime}-v^{\prime \prime}=0
$$

Now integrate equation (3.3) with respect to $\eta$.

$$
v^{\prime \prime \prime}+3\left(v^{\prime}\right)^{2}+(c-1) v^{\prime}=0
$$

Now by using homogenous balance method we find $N=2$.

In the third step substitute the derivatives of function $v(\eta)$ into equation (3.4). In this case these derivatives can be written as

$$
\begin{aligned}
v_{\eta \eta \eta} & =a_{1} Q(Q-1)\left(6 Q^{2}-6 Q+1\right)+2 a_{2} Q^{2}(Q-1)\left(12 Q^{2}-15 Q+4\right) \\
v_{\eta \eta} & =a_{1} Q(Q-1)[2 Q-1]+2 a_{2} Q^{2}(Q-1)[(3 Q-2)] \\
v_{\eta} & =a_{1} Q(Q-1)+2 a_{2} Q^{2}(Q-1)
\end{aligned}
$$

the expression $v(\eta)$ in the form

$$
v=a_{0}+a_{1} Q+a_{2} Q^{2}
$$

As result of the third step we have the following equation

$$
\begin{aligned}
& 12 a_{2}{ }^{2} Q^{6}+\left(12 a_{1} a_{2}-24 a_{2}{ }^{2}+24 a_{2}\right) Q^{5}+\left(6 a_{1}-54 a_{2}-24 a_{1} a_{2}+3 a_{1}{ }^{2}+12 a_{2}{ }^{2}\right) Q^{4}+ \\
& \left(-12 a_{1}+12 a_{1} a_{2}+36 a_{2}+2 c a_{2}-6 a_{1}{ }^{2}\right) Q^{3}+\left(-6 a_{2}-2 c a_{2}+c a_{1}+3 a_{1}{ }^{2}+6 a_{1}\right) Q^{2}-c a_{1} Q=0
\end{aligned}
$$

4. Now equate terms of equation (3.9) equal to zero

$$
\begin{aligned}
& 12 a_{2}{ }^{2}=0 \\
& \left(12 a_{1} a_{2}-24 a_{2}^{2}+24 a_{2}\right)=0 \\
& \left(6 a_{1}-54 a_{2}-24 a_{1} a_{2}+3 a_{1}^{2}+12 a_{2}^{2}\right)=0 \\
& \left(-12 a_{1}+12 a_{1} a_{2}+36 a_{2}+2 c a_{2}-6 a_{1}^{2}\right)=0 \\
& \left(-6 a_{2}-2 c a_{2}+c a_{1}+3 a_{1}^{2}+6 a_{1}\right)=0 \\
& -c a_{1}=0
\end{aligned}
$$

Solving the system of equations by Maple Software

Case $1 a_{1}=0=a_{2}, c=c$

Case $2 a_{1}=-2, a_{2}=0=c$

6. Analytical solutions of the SWW equation take the form

$$
v(\xi)=a_{0}+a_{1}\left(\frac{1}{1+e^{\eta}}\right)+a_{2}\left(\frac{1}{1+e^{\eta}}\right)
$$

Where $\eta=x+y+z-c t$.

\section{References}

1. Anjali Verma, Ram Jiwari and Jitender Kumar, "Traveling Wave solutions for shallow water wave equation by $\left(G^{\prime} / G\right)$-expansion method". International Journal of Mathematical Sciences. 7(1) 2013, pp.12-16.

2. A.A Imani, D.D Ganji, Houman B. Rokni, H. Latifizadeh, Esmail Hesameddini, M. Hadi Rafiee, “ Approximate travelling wave solution for shallow water wave equation”. Applied Mathematical Modelling. 36 (2012), pp. 1550-1557. 
3. Ahmet Bekir and Esin Aksoy, “ Exact solutions of shallow water equation by using (G'/G)-expansion method. Waves in reandom and complex Media. Vol. 22, 2012.

4. B. Aydm and U. Kanoglu, "Analytical solutionfor nonlinear shallow water wave equation." 15 WCEE LIS BOA 2012.

5. Bijan Bagchi, Supratim Das and Asish Ganguly, "New exact solutions of a generalized shallow water wave equation.” Physica Scripta. 2010.

6. Elizabeth L. Mansfield and Peter A. Clarkson, "Symmetries and exact solutions for $(2+1)$-dimensional shallow water wave equation. Mathematics and Computers in Simulation. Vol. 43 (1997) pp. 39-55.

7. N. A. Matskevich and L. B. Chubarov, "Exact solutions to shallow water equation for a water oscillation problem in idealized Basin and their use in verifying some Numerical Algorithms." Numerical Analysis and Applications. Vol. 12 (2019) pp. 234-250.

8. Peter A. Clarkson and Elizabeth L. Mansfield, "Symmetry reduction and exact solutions of shallow water wave equation.” Acta Applicandae Mathematica. 2008.

9. Wiiliam Carlisle Thacker, " Some exact solutions to the nonlinear shallow water wave equation." Combridge University Press. Vol. 107 (1981) pp. 499-508. 